RESEARCH REPORT

\title{
Is the rate of biological aging, as measured by age at diagnosis of cancer, socioeconomically patterned?
}

\author{
Jean Adams, Martin White, David Forman
}

J Epidemiol Community Health 2005;59:146-151. doi: 10.1136/jech.2004.021006

\begin{abstract}
See end of article for authors' affiliations

.....

Correspondence to: Dr J Adams, School of Population and Health Science, The Medical School, University of Newcastle upon Tyne, Newcastle upon Tyne NE2 $4 \mathrm{HH}$, UK; j.m.adams@nd. ac.uk
\end{abstract}

Accepted for publication 22 May 2004

\begin{abstract}
Study objective: To investigate the hypothesis that biological aging, as measured by age at diagnosis of some common cancers, is socioeconomically patterned.

Design: A cross sectional analysis of the association between an area based measure of material deprivation and age at diagnosis of four common cancers (breast, prostate, colorectal, and lung cancers). A further analysis, restricted to breast and colorectal cancer, adjusted for stage and grade of cancer at diagnosis.

Setting: The Northern and Yorkshire cancer registry and information service, Northern and Yorkshire region, UK.

Participants: All people living in the Northern and Yorkshire region diagnosed with breast, prostate, colorectal, or lung cancer in 1986-1995. All people living in the Northern and Yorkshire region diagnosed with breast or colorectal cancer in 1998-2000 with data on stage and grade of cancer at diagnosis.

Main results: There was evidence that greater material deprivation was associated with younger age at diagnosis of cancer in prostate ( $\beta$ coefficient -0.073 ), colorectal (women: -0.042 ; men: -0.063 ), and lung cancer (women: -0.214 ; men: -0.161 ). The opposite association was found in women with breast cancer (0.149). Adjusting for stage and grade at incidence, where possible, had little effect on the magnitude of the $\beta$ coefficients.

Conclusions: Age at diagnosis of some common cancers seems to be socioeconomically patterned with people from more deprived areas being diagnosed with prostate, colorectal, and lung cancers earlier in life. The opposite was seen in women with breast cancer. Further work is required to investigate the socioeconomic distribution of more accurate measures of biological aging.
\end{abstract}

Cellular defence and repair mechanisms include antioxidant vitamins and enzymes and DNA checking and repair enzymes.

Unlike chronological aging, there is considerable variation between individuals in the rate of biological aging. ${ }^{12}$ Both genetic and environmental factors play a part $^{13}{ }^{14}$ and numerous environmental factors, known to be socioeconomically patterned, are now known to influence the rate of cellular damage accumulation, including cigarette smoking, ${ }^{15}$ diet, ${ }^{16}$ and exposure to radiation. ${ }^{17}$

Furthermore, there is good reason to believe that biological aging and the accumulation of cellular damage are important determinants of health. Population based research suggests that some crude markers of aging, such as total and healthy life expectancy, are socioeconomically patterned. ${ }^{18} 19$ However, no research, to date, has attempted to investigate the socioeconomic distribution of more accurate measures of biological aging. Cancers are attributable, at the most proximate level, to acquired or inherited mutations in genes that control growth-specifically, oncogenes and tumour suppressor genes. ${ }^{20}$ As genetic mutations are one, well recognised, type of cellular damage, ${ }^{11}$ the development of cancer can be understood as being closely related to the processes involved in biological aging. Chronological age of development of cancer can, therefore, be used as a comparative measure of biological aging.

Abbreviations: SEP, socioeconomic position; SEDH, socioeconomic differences in health; NYCRIS, Northern and Yorkshire Cancer Registration and Information Service rant proteins within the cytosol, reactive oxygen species, and the oxidative stress they generate and somatic mutations. ${ }^{11}$ 


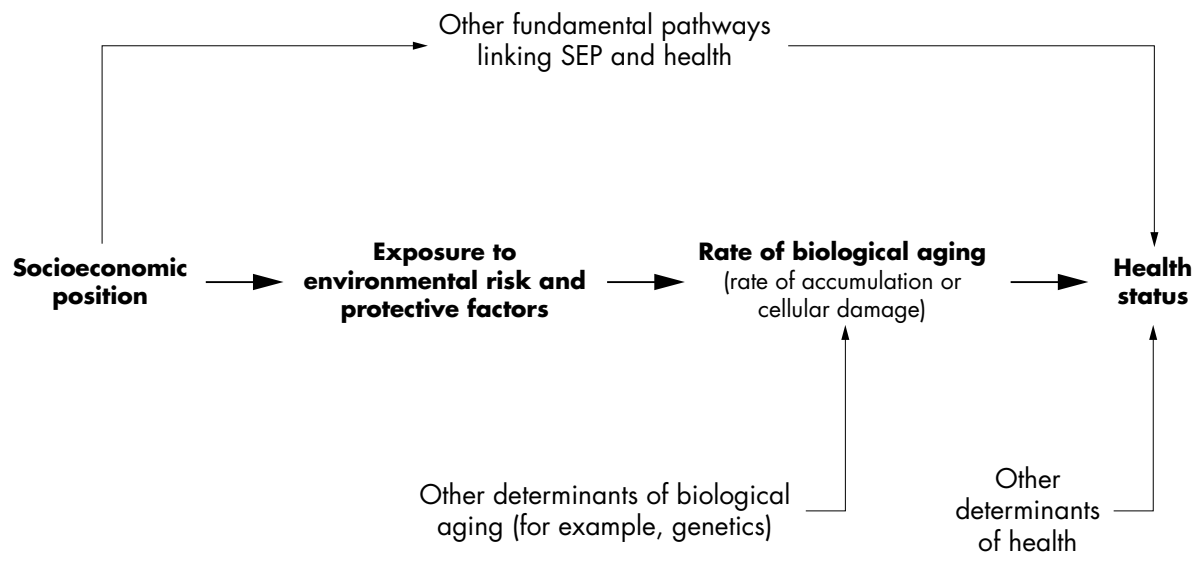

Figure 1 The hypothesised pathway linking socioeconomic position, environmental risk and protective factors, biological aging, and health status.
We investigated the effect of SEP on the age of development of some common cancers using data from a population based cancer registry.

\section{METHODS}

The Northern and Yorkshire Cancer Registration and Information Service (NYCRIS) is one of nine regional cancer registries in England that aims to collect data on all incident cancers as they occur. We used data held by NYCRIS to investigate the effects, if any, of SEP on age at diagnosis of cancer.

\section{Variables of interest}

SEP was measured using Townsend deprivation scores $(\mathrm{TDS})^{4}$ of the enumeration district of residence at date of diagnosis of cancer calculated from full seven digit postcode data and standardised to the NYCRIS region as a whole using data from the 1991 census. A positive TDS represents more material deprivation while a negative score represents less material deprivation.

Age at diagnosis of cancer was calculated from incident date and date of birth. As is routine in UK cancer registries, incident date was defined as the first available (not necessarily earliest) date from: date of pathologically confirmed diagnosis, date of first hospital attendance during which a clinical diagnosis was made, date of diagnosis by a $\mathrm{GP}$, and date of death.

Cancer site was assigned using three figure codes from the 10th revision of the International Classification of Diseases (ICD-10) as extrapolated from clinical records and recorded in the NYCRIS database. We restricted the analysis to the four commonest non-skin cancer sites: breast (ICD-10 C50), colorectal (ICD-10 C18, C19 or C20), lung (ICD-10 C33 or C34), and prostate cancer (ICD-10 C61).

\section{Sample size and study years}

As no previous work in this area has been reported, we were unable to perform sample size or power calculations. Instead, we adopted a pragmatic approach aiming to use as large a sample size as possible. This was, however, restricted by our measure of SEP, TDS, which are calculated from decennial UK census data. To avoid using data from more than one census, we restricted ourselves to 10 years of NYCRIS data and chose to centre our data collection years around a census year so that the maximum temporal extrapolation of census data would be five years. The most recent census year for which data could be extrapolated five years both backwards and forwards was 1991. We therefore chose 1986-1995 inclusive as our study years.

\section{Further analysis to assess the effects of adjusting for stage and grade at diagnosis}

Using the method described to determine incident date, and therefore age at diagnosis, does not necessarily result in comparable data for all individuals. To minimise any bias or error introduced by this method, we wished to adjust for stage and grade of cancer at diagnosis. However, these data were far from complete for the main study cohort and it was estimated that the only cohorts with more than $50 \%$ complete data on stage and grade were individuals registered with colorectal and breast cancers since 1998. We, therefore, performed a further analysis, using similar methods as the main study, which investigated the effect of adjusting for stage and grade at diagnosis on the relation between age and TDS at diagnosis. This analysis used data on colorectal and breast cancers registered between 1998 and 2000 inclusive.

\section{Exclusions}

People were excluded from the analysis if: key data were missing, registration had been by death certification only

Table 1 Distribution of age and Townsend deprivation score at diagnosis of cancer (1986-1995 cohort)

\begin{tabular}{|c|c|c|c|c|c|c|c|c|c|c|}
\hline & \multicolumn{5}{|c|}{ Before exclusion of youngest $25 \%$ at diagnosis } & \multicolumn{5}{|c|}{ After exclusion of youngest $25 \%$ at diagnosis } \\
\hline & \multirow[b]{2}{*}{ Number } & \multicolumn{2}{|l|}{ Age } & \multicolumn{2}{|l|}{ TDS } & \multirow[b]{2}{*}{ Number } & \multicolumn{2}{|l|}{ Age } & \multicolumn{2}{|l|}{ TDS } \\
\hline & & Median & IQR & Median & IQR & & Median & IQR & Median & IQR \\
\hline Prostate cancer & 17107 & 74.80 & 68.89 to 80.47 & -0.51 & -2.66 to 2.27 & 12828 & 77.63 & 73.48 to 82.11 & -0.35 & -2.58 to 2.40 \\
\hline Breast cancer, women & 34265 & 62.63 & 51.44 to 73.67 & -0.57 & -2.73 to 2.23 & 25697 & 67.84 & 60.09 to 76.92 & -0.50 & -2.65 to 2.19 \\
\hline Colorectal cancer, women & 16872 & 74.29 & 65.65 to 81.62 & -0.11 & -2.43 to 2.68 & 12656 & 78.05 & 72.41 to 83.53 & 0.03 & -2.27 to 2.71 \\
\hline Colorectal cancer, men & 18053 & 70.30 & 62.78 to 77.28 & -0.02 & -2.46 to 2.81 & 13538 & 73.67 & 68.51 to 79.29 & 0.06 & -2.36 to 2.81 \\
\hline Lung cancer, women & 18116 & 69.78 & 63.23 to 77.28 & 1.36 & -1.38 to 3.72 & 14165 & 72.46 & 67.65 to 78.14 & 1.28 & -1.38 to 3.64 \\
\hline Lung cancer, men & 35273 & 70.03 & 63.75 to 76.31 & 1.23 & -1.50 to 3.66 & 26442 & 72.98 & 68.58 to 78.24 & 1.09 & -1.55 to 3.54 \\
\hline
\end{tabular}


Table 2 Linear regression models of the ability of Townsend deprivation score to predict age at diagnosis of cancer (19861995 cohort)

\begin{tabular}{|c|c|c|c|c|c|c|}
\hline & $\beta$ coefficient & $95 \% \mathrm{Cl}^{*}$ & $t$ Test & $p$ value & Adjusted $r^{2}$ & $\begin{array}{l}\text { Change in age }(y) / \\
\text { TDS IQR† }\end{array}$ \\
\hline Prostate cancer & -0.073 & -0.106 to -0.041 & -4.446 & $<0.0001$ & 0.001 & -0.36 \\
\hline Breast cancer & 0.149 & 0.109 to 0.193 & 7.018 & $<0.0001$ & 0.002 & 0.72 \\
\hline Colorectal cancer, women & -0.042 & -0.081 to -0.004 & -2.070 & 0.039 & $<0.001$ & -0.21 \\
\hline Colorectal cancer, men & -0.063 & -0.100 to -0.027 & -3.367 & 0.001 & 0.001 & -0.33 \\
\hline Lung cancer, women & -0.214 & -0.252 to -0.177 & -11.350 & $<0.0001$ & 0.009 & -1.07 \\
\hline Lung cancer, men & -0.161 & -0.185 to -0.136 & -12.886 & $<0.0001$ & 0.006 & -0.82 \\
\hline
\end{tabular}

*95\% Confidence intervals (bias corrected) of $\beta$ derived from bootstrapping with 1000 repetitions; $†$ change in age at diagnosis of cancer across the interquartile range in Townsend deprivation score from lower (most affluent) quartile to upper (most deprived quartile).

(and therefore the date of incidence had been recorded as the date of death), ${ }^{21}$ or the incident cancer was a second primary. Men with breast cancer were excluded because of the rarity of this condition.

A proportion of individuals inherit a specific genetic defect that puts them at high risk of developing a cancer early in life. ${ }^{22}$ We felt that such individuals formed a distinct group and should not be included in the present analyses because of our focus on environmental factors. As cancer registries do not routinely record information on inherited susceptibilities to cancer, we excluded the youngest $25 \%$ of individuals, at diagnosis, from each cancer and gender specific group as a conservative approach to removing all those whose cancer may include a substantial inherited component.

\section{Analysis}

Data were analysed in gender and cancer site specific groups throughout. Linear regression techniques were used to assess the ability of TDS at diagnosis to predict age at diagnosis of cancer both before, and in the case of the 1998-2000 cohort, after adjustment for stage and grade of cancer at diagnosis. Neither age nor TDS was normally distributed in any of the gender and cancer site specific groups. As simple transformation did not result in appreciably more normal distributions of these variables, bootstrapping methods, with 1000 repetitions, were used to generate confidence intervals for $\beta$ coefficients derived from linear regression techniques. ${ }^{23}$ All analyses were performed in Intercooled Stata version 8.0.

\section{RESULTS}

\section{The 1986-1995 cohort}

Between I January 1986 and 31 December 1995, 144627 cases of lung, colorectal, prostate, and breast cancer were registered with NYCRIS. Of these, $39301(27.2 \%)$ met one of the exclusion criteria, including the age cut off, leaving 105326 individuals in the analysis.

Table 1 shows the distribution of age and TDS at diagnosis in the 1986-1995 cohort before and after exclusion of the youngest $25 \%$ of individuals at diagnosis. In the cohort included in the analysis, women with breast cancer were youngest (median age 67.84) and women with colorectal cancer oldest (median age 78.05) at diagnosis. In addition, women with breast cancer were least deprived (median TDS -0.50 ) and women with lung cancer most deprived (median TDS 1.28) at diagnosis. While exclusion of the youngest 25\% at diagnosis led to an overall increase in the affluence of the cohorts with lung cancer, the opposite was seen in the other groups.

Table 2 shows the results of simple linear regression analysis of the ability of TDS at diagnosis to predict age at diagnosis. The $95 \%$ confidence intervals of the $\beta$ coefficients of TDS at diagnosis excluded unity in all cases. The negative $\beta$ coefficients seen in relation to prostate ( $\beta$ coefficient -0.073 ), colorectal (women: -0.042 ; men: -0.063 ), and lung cancer (women: -0.214 ; men: -0.161 ) suggest that people living in more deprived areas tended to have these cancers diagnosed earlier in life than those living in more affluent areas. These coefficients equate to a variation in age at diagnosis of cancer of between 0.21 (colorectal cancer, women) and 1.07 years (lung cancer, women) across the interquartile range of TDS.

Among women with breast cancer, the $\beta$ coefficient of TDS at diagnosis was positive (0.149) suggesting that women living in more deprived areas tended to have breast cancer diagnosed later in life than those living in more affluent areas. This equates to a variation of 0.72 years in age at diagnosis across the interquartile range of TDS.

\section{The 1998-2000 cohort}

There were 25314 cases of colorectal and breast cancer registered with NYCRIS between 1 January 1998 and 31 December 2000. Of these, 11445 (45.2\%) were excluded from the analysis-almost half of these exclusions were made on the grounds of missing stage or grade data. There was evidence that those excluded from the analysis on the grounds of missing stage or grade data were from more deprived neighbourhoods than those included $(\mathrm{p}<0.0001)$ and were older $(\mathrm{p}<0.0001)$. Table 3 shows the distribution of

Table 3 Distribution of age and Townsend deprivation score at diagnosis of cancer (1998-2000 cohort)

\begin{tabular}{|c|c|c|c|c|c|c|c|c|c|c|}
\hline & \multicolumn{5}{|c|}{ Before exclusion of youngest $25 \%$ at diagnosis } & \multicolumn{5}{|c|}{ After exclusion of youngest $25 \%$ at diagnosis } \\
\hline & \multirow[b]{2}{*}{ Number } & \multicolumn{2}{|l|}{ Age } & \multicolumn{2}{|l|}{ TDS } & \multirow[b]{2}{*}{ Number } & \multicolumn{2}{|l|}{ Age } & \multicolumn{2}{|l|}{ TDS } \\
\hline & & Median & IQR & Median & IQR & & Median & IQR & Median & IQR \\
\hline $\begin{array}{l}\text { Breast cancer, } \\
\text { women }\end{array}$ & 9920 & 58.50 & 50.18 to 69.21 & -1.04 & -2.99 to 1.79 & 7441 & 63.30 & 55.99 to 72.63 & -1.04 & -3.00 to 1.82 \\
\hline $\begin{array}{l}\text { Colorectal cancer, } \\
\text { women }\end{array}$ & 3707 & 72.67 & 64.54 to 79.45 & -0.54 & -2.74 to 2.44 & 2781 & 76.11 & 70.86 to 81.46 & -0.39 & -2.65 to 2.51 \\
\hline $\begin{array}{l}\text { Colorectal cancer, } \\
\text { men }\end{array}$ & 4861 & 70.38 & 62.68 to 76.62 & -0.72 & -2.78 to 2.21 & 3647 & 73.71 & 68.72 to 78.30 & -0.64 & -2.74 to 2.26 \\
\hline
\end{tabular}


Table 4 Linear regression models of the ability of Townsend deprivation score to predict age at diagnosis of cancer before and after adjustment for, stage and grade at diagnosis (1998-2000 cohort)

\begin{tabular}{|c|c|c|c|c|c|c|c|c|c|c|c|c|}
\hline & \multicolumn{6}{|c|}{ Unadjusted } & \multicolumn{6}{|c|}{ Adjusted for stage and grade at diagnosis of cancer } \\
\hline & $\beta$ & $95 \% \mathrm{Cl}^{*}$ & $t$ Test & $\mathrm{p}$ Value & Adjusted $r^{2}$ & $\begin{array}{l}\text { Change in age } \\
\text { (y)/TDS IQR† }\end{array}$ & $\beta$ & $95 \% \mathrm{Cl}^{*}$ & $t$ Test & $p$ Value & Adjusted $r^{2}$ & $\begin{array}{l}\text { Change in age } \\
\text { (y)/TDS IQRT }\end{array}$ \\
\hline Breast cancer & 0.227 & 0.159 to 0306 & 6.069 & $<0.0001$ & 0.005 & 1.09 & 0.205 & 0.127 to 0.285 & 5.533 & $<0.0001$ & 0.021 & 0.99 \\
\hline $\begin{array}{l}\text { Colorectal cancer, } \\
\text { women }\end{array}$ & 0.009 & -0.074 to 0.096 & 0.151 & 0.880 & $<0.0001$ & 0.05 & 0.012 & -0.073 to 0.088 & 0.226 & 0.821 & $<0.0001$ & 0.06 \\
\hline $\begin{array}{l}\text { Colorectal cancer, } \\
\text { men }\end{array}$ & -0.056 & -0.119 to 0.012 & -1.617 & 0.106 & $<0.0001$ & -0.28 & -0.055 & -0.128 to 0.011 & -1.572 & 0.116 & $<0.0001$ & 0.28 \\
\hline
\end{tabular}

age and TDS at incidence in the 1998-2000 cohort before and after exclusion of the youngest $25 \%$ of individuals at diagnosis. As before, women with breast cancer tended to be youngest (median age 63.30) and women with colorectal cancer tended to oldest (median age 76.11) at diagnosis. Individuals included in the 1998-2000 cohort tended to be more affluent and slightly younger at cancer incidence than those in comparable groups in the main 1986-1995 cohort.

Table 4 shows the results of linear regression analyses of the ability of TDS at diagnosis to predict age at diagnosis both before and after adjustment for stage and grade at diagnosis. There was less evidence of statistically significant relations between TDS and age at diagnosis in this cohort compared with the 1986-1995 cohort. However, adjustment for stage and grade at diagnosis had little effect on the magnitude of the $\beta$ coefficients in all three site and gender specific groups in the 1998-2000 cohort.

\section{DISCUSSION}

This is the first study that has assessed the socioeconomic distribution of either age at diagnosis of cancer or other possible markers of biological aging. Using data from more than 100000 people, we found evidence of a small, negative, effect of TDS on age at diagnosis of prostate, lung, and colorectal cancers. People living in more materially deprived areas tended to have these cancers diagnosed earlier in life with a difference of between 0.21 and 1.07 years in age at diagnosis of cancer across the interquartile range of TDS. The opposite relation was found among women with breast cancer with women living in more deprived areas tending to have breast cancer diagnosed later in life than those living in less deprived areas. In a second analysis, adjusting for stage and grade of cancer at incidence had very little effect on the magnitude of the effect of TDS on age at diagnosis for breast and colorectal cancers.

Although routinely collected data, such as used here, are not always of high quality, there is reason to believe that the UK cancer registries, and NYCRIS in particular, collect data of a consistently high standard. ${ }^{21}$ For example, in 1994 NYCRIS collected contemporaneous data from more than $93 \%$ of

\section{Key points}

- Rate of biological aging may mediate the relation between socioeconomic position and health and age at diagnosis of cancer may be a comparative marker of biological aging with earlier age at diagnosis being indicative of faster biological ageing.

- More deprived people tended to develop colorectal, lung, and prostate cancer earlier in life than more affluent people. The opposite trend was seen in relation to breast cancer. people who died with cancer in the region-compared with a UK average of only $88 \% .{ }^{24}$ In addition, only $497(0.3 \%$ of the total number of registrations) people were excluded from the main cohort in this study because of missing data. We are, therefore, confident that our analysis, at least of the main 1986-1995 cohort, used high quality data with good ascertainment and completeness. In contrast, substantial exclusions were made from the 1998-2000 cohort because of missing data on stage or grade of cancer. We are, therefore, less confident of the quality of these data and cannot be sure that adjusting for stage and grade of cancer in this cohort provided us with a clear picture of actual effect of this procedure in a more complete cohort.

It is not necessarily desirable or appropriate to apply area based measures of SEP, such as TDS, to individuals as done here. The process entails both the assumption that small areas are comparatively socioeconomically homogeneous and significant temporal extrapolation of data. ${ }^{25}{ }^{26}$ However, the limitations of the data collected by NYCRIS meant that an area based measure of SEP was all that was available to us. A wide variety of previous work has applied TDS to individuals $^{27-31}$ and we made the pragmatic decision to use TDS as an individual measure of SEP to make use of this large and rich dataset.

We used age at diagnosis of cancer as a proxy for age at development of cancer. Any socioeconomic variations in the length of time between cancer development and diagnosis therefore have the potential to confound the relations seen. However, the research evidence concerning socioeconomic variations in diagnostic delay is far from consistent ${ }^{32-35}$ and adjustment for stage and grade at incidence (two key markers of cancer progression and, therefore, diagnostic delay) in this study had very little effect on the magnitude of the effect of TDS on age at diagnosis, suggesting that variations in timeliness of diagnosis did not explain the results seen. Given the quality of these data we cannot, however be confident of this conclusion. However, recent research has reported an inverse association between deprivation and proximity to health services ${ }^{36}$ suggesting that more deprived individuals are actually more likely to live in close proximity to treatment centres than more affluent ones. It is hard to define, even theoretically, exactly when a cancer occurs. Using the date at which a cancer is clinically detectable is one possible method but this would require a

\section{Policy implications}

If the rate of biological aging is confirmed as mediating the relation between socioeconomic position and health, targeting interventions that reduce the rate of biological aging at deprived populations may help reduce socioeconomic variations in health. 
large prospective screening study with a short time span between screens. Our analyses have provided preliminary data in this area without recourse to such a lengthy and resource intensive study.

In addition to using date of diagnosis as a proxy for date of development of cancer, we also used it as a proxy for biological aging. Although we believe that age at diagnosis of cancer is a useful comparative clinical marker of the rate of biological ageing, the pathways between biological aging and diagnosis of cancer are long and complex and the cellular damage responsible for cancer is not necessarily the same cellular damage responsible for biological aging. The results presented here can only begin to untangle the relations, if any, between SEP and biological aging.

Because our hypothesis focuses on environmental risk and protective factors, we felt that it was important to exclude individuals with cancers that had a substantial inherited component. This was done, somewhat arbitrarily, by excluding the youngest $25 \%$ of each gender and site specific group on the basis that inherited cancers are likely to occur earlier in life than sporadic ones, with inevitable knock on effects on the age and TDS distributions of the cohorts used in the analyses. ${ }^{22}$ However, the division between cancers with a large inherited component and those that are primarily sporadic is not clear cut and not all of the $25 \%$ of cancers occurring at the youngest ages will necessarily have a predominant inherited component, nor will all cancers with a strong inherited component necessarily occur within this age group.

Furthermore, despite the statistical significance of the influence of TDS on age at diagnosis found, the effect sizes seen were small with a standard deviation change in TDS at diagnosis resulting in less than a year change in age at diagnosis of cancer. The inaccuracies in our measure of biological aging and the effects of unknown confounders may contribute to the minimal effect size seen and further research investigating socioeconomic variations of more accurate markers of biological aging is warranted.

We have identified two possible explanations for the main finding that age at diagnosis of prostate, colorectal, and lung cancer decreases with increasing deprivation of the area of residence. Firstly, it is possible that the cancers that individuals across the socioeconomic range develop are biologically different in some way that leads to variations in age of onset. It has been highlighted that not all cancers, even of the same tissue, involve the same disease process. ${ }^{37}$ Given the different environmental risks and hazards, and therefore carcinogens, that individuals across the socioeconomic range are exposed to, it is possible that, despite being classified under the same ICD heading in the NYCRIS database, not all cancers that we have analysed are comparable. Although such biological variations have been recorded according to age,,$^{38-40}$ we are not aware of any evidence that the biological nature of individual cancer types varies by SEP. An alternative explanation of our findings is that the rate of accumulation of damage at the cellular level, and thus biological ageing, is socioeconomically patterned due to socioeconomic patterning of exposure to carcinogenic risk factors (fig 1).

Neither of the preceding explanations can be applied to the results seen among women with breast cancer. The one key difference in the diagnosis of breast cancer in British women, compared with the other cancers studied, is the presence of the UK national breast cancer screening programme. This programme invites all women aged between 50 and 64 for triennial mammography. Although highly successful in detecting early breast cancer and reducing morbidity and mortality, ${ }^{41}$ uptake of breast screening in the UK is known to be socioeconomically patterned with more affluent women tending to attend more regularly than less affluent women. ${ }^{42}{ }^{43}$ It is, therefore, possible that the tendency we found for more affluent women to have breast cancer diagnosed at younger ages is attributable to their more regular attendance for breast cancer screening and resultant more timely diagnosis of breast cancer. As we did not have information of who did and did not attend for screening, we were unable to explore this possibility further. However, the plausibility of this explanation further calls into question the accuracy of our adjustment for stage and grade of cancer at diagnosis in the 1998-2000 cohort.

\section{Conclusions}

By providing evidence that the age at diagnosis of some common cancers is socioeconomically patterned, this study provides preliminary evidence that the rate of biological aging may also be socioeconomically patterned. Furthermore, we found little evidence that socioeconomic variations in diagnostic delay-as measured by stage or grade of cancer at diagnosis-were confounding the relations seen. To further investigate the relation between SEP and biological aging, studies using longitudinal measures of individual socioeconomic circumstances across the life course and measures of the actual process of biological aging at the cellular level, rather than the clinical outcomes of it, will be required.

\section{ACKNOWLEDGEMENTS}

The authors thank Caroline Brook and Cheryl Craigs at NYCRIS for methodological suggestions and help with abstracting data and Nigel Unwin at Newcastle University for critical comments on a previous draft of the manuscript.

\section{Authors' affiliations}

J Adams, M White, School of Population and Health Science, The Medical School, University of Newcastle upon Tyne, UK

D Forman, Northern and Yorkshire Cancer Registry and Information Service, Cookridge Hospital, Leeds, UK

Funding: JA is supported by the BUPA/Faculty of Public Health Medicine Joint Research Fellowship (2001-2004).

Conflicts of interest: none declared.

\section{REFERENCES}

1 Whitehead M. Inequalities in health: the health divide. 3rd ed. London: Penguin Books, 1992.

2 Acheson D. Report of the independent enquiry into inequalities in health. London: Stationery Office, 1998.

3 Townsend P, Davidson N, eds. Inequalities in health: the Black report. London: Penguin Books, 1982.

4 Townsend P, Phillimore P, Beattie A. Health and deprivation: inequality and the North. Bristol: Croom Helm, 1988.

5 Charlton B, White M. Living on the margin: a salutogenic model for socioeconomic differentials in health. Public Health 1995; 109:235-43.

6 Fuhrer R, Shipley M, Chastang J, et al. Socioeconomic position, health and possible explanations: a tale of two cohorts. Am J Public Health 2002;92:1290-4

7 Adams J, White M. Biological ageing: A fundamental, biological link between socio-economic status and health? Eur J Public Health 2004; 14:31-4.

8 Germov J, ed. Second opinion: an introduction to health sociology. Singapore: Oxford University Press, 1998.

9 Fries J, Crapo L. Vitality and aging: implications of the rectangular shaped curve. San Franciso: WH Freeman and Company, 1981.

10 Kirkwood T, Austad S. Why do we age? Nature 2000;408:233-8.

11 Kirkwood T, Kowald A. Network theory of ageing. Exp Gerontol 1997;32:395-9.

12 Wick G, Jansen-Durr $P$, Berger $P$, et al. Diseases of aging. Vaccine 2000;18:1567-83.

13 Guarente L, Kenyon C. Genetic pathways that regulate ageing in model organisms. Nature 2000;408:255-62.

14 Cournil A, Kirkwood T. If you would live long, choose your parents well. Trends Genet 2001;17:233-5.

15 MacNee W. Oxidants/antioxidants and COPD. Chest 2000;117(suppl 1):303-17S

16 Taylor A, Jaques P, Epstein E. Relations among aging, antioxidant status, and cataract. J Clin Nutr 1995;62(suppl): 1439-47s.

17 Young R. Age-related cataract. New York: Oxford University Press, 1991. 
18 Drever F, Whitehead M, eds. Health inequalities decennial supplement. London: The Stationery Office, 1997.

19 Crimmins E, Saito Y. Trends in healthy life expectancy in the United States, 1970-1990: gender, racial, and educational differences. Soc Sci Med 2001:52:1629-41.

20 Bishop J, Weinberg R, eds. Molecular oncology. New York: Scientific American, 1996.

21 Hugget C. Nationwide audit of the quality and comparability of data held by regional cancer registries. Bristol: University of Bristol, 1995.

22 Lindblom A, Nordenskjold M. The biology of inherited cancer. Semin Cancer Biol 2000; 10:251-4.

23 Kirkwood B, Sterne J. Essential medical statistics. 2nd ed. Bodmin: Blackwell Science, 2003.

24 Quinn M, Babb P, Brock A, et al. Cancer trends in England and Wales 19501999. London: The Stationery Office, 2001.

25 Krieger N, Williams D, Moss N. Measuring social class in US public health research: concepts, methodologies and guidelines. Annu Rev Public Health 1997; 18:341-78.

26 Carr-Hill R, Chalmers-Dixon P. A review of methods for monitoring and measuring social inequality, deprivation and health inequality. York: University of York, 2002.

27 Taylor A, Cheng K. Social deprivation and breast cancer. J Public Health Med 2002;25:228-33.

28 McNally R, Alston R, Cairns D, et al. Geographical and ecological analyses of childhood acute leukaemias and lymphomas in north-west England. Br J Haematol 2003;123:60-5.

29 Bundred $P$, Manning D, Brewster B, et al. Social trends in singleton births and birth weight in Wirral residents, 1990-2001. Arch Dis Child Fetal Neonatal Ed 2003:88:F421-4.

30 Feltbower R, McKinney P, Campbell F, et al. Type 2 and other forms of diabetes in 0-30 year olds: hospital based study in Leeds, UK. Arch Dis Child 2003;88:676-9.

31 Aggarwal V, Macfarlane T, Macfarlane G. Why is pain more common amongst people living in areas of low socio-economic status? A populationbased cross-sectional study. Br Dent J 2003;194:383-7.
32 Macleod U, Ross S, Gillis C, et al. Socio-economic deprivation and stage of disease at presentation in women with breast cancer. Ann Oncol 2000;11:105-7.

33 Thomson C, Hole D, Twelves C, et al. Prognostic factors in women with breast cancer: distribution by socioeconomic status and effect on difference in survival. J Epidemiol Community Health 2001;55:308-15.

34 Brewster D, Thomson C, Hole D, et al. Relation between socioeconomic status and tumour stage in patients with breast, colorectal, ovarian, and lung cancer: results from four national, population based studies. BMJ 2001;322:830-1.

35 Carnon A, Ssemwogerere A, Lamont D, et al. Relation between socioeconomic deprivation and pathological prognostic factors in women with breast cancer. BMJ 1994;309:1054-7.

36 Jordan H, Roderick P, Martin D. The index of multiple deprivation 2000 and accessibility effects on health. J Epidemiol Community Health 2004;58:250-7.

37 Macieira-Coelho A. Neoplastic disease through the human life span. Biogerontology 2001 ; 2:179-92.

38 Awadh-Behbehani M, Al-Humood K, Ayed A, et al. Comparison between young and old patients with bronchogenic carcinoma. Acta Oncol 2000;39:995-9.

39 Mettlin C, Murphy G, Cunningham M, et al. The national cancer data base report on race, age, and region variations in prostate cancer treatment. Cancer 1997;80:1261-6.

40 Erdreich L, Asal N, Hoge A. Morphologic types of breast cancer: age, bilaterality and family history. South Med J 1980;73:28-32.

41 Blanks R, Moss S, McGahan C, et al. Effect of NHS breast screening programme on mortality from breast cancer in England and Wales, 1990-8: comparison of observed with predicted mortality. BMJ 2000;321:665-9.

42 Sutton S, Bickler G, Sancho-Aldridge J, et al. Prospective study of predictors of attendance for breast screening in inner London. J Epidemiol Community Health 1994;48:65-73.

43 Garvican L, Littlejohns P. Comparison of prognostic and socio-economic factors in screen-detected and symptomatic cases of breast cancer. Public Health 1998;112:15-20. 